\title{
Subcortical Modulation of Attention Counters Change Blindness
}

\author{
James Cavanaugh and Robert H. Wurtz \\ Laboratory of Sensorimotor Research, National Eye Institute, National Institutes of Health, Bethesda, Maryland 20817
}

\begin{abstract}
Change blindness is the failure to see large changes in a visual scene that occur simultaneously with a global visual transient. Such visual transients might be brief blanks between visual scenes or the blurs caused by rapid or saccadic eye movements between successive fixations. Shifting attention to the site of the change counters this "blindness" by improving change detection and reaction time. We developed a change blindness paradigm for visual motion and then showed that presenting an attentional cue diminished the blindness in both humans and old world monkeys. We then replaced the visual cue with weak electrical stimulation of an area in the monkey's brainstem, the superior colliculus, to see if activation at such a late stage in the eye movement control system contributes to the attentional shift that counters change blindness. With this stimulation, monkeys more easily detected changes and had shorter reaction times, both characteristics of a shift of attention.
\end{abstract}

Key words: attention; change blindness; motion; saccades; superior colliculus; stimulation; visual

\section{Introduction}

Visual transients capture attention. When a focal visual transient occurs, such as a small feature of a scene suddenly appearing or changing, the viewer's attention shifts to it (Yantis and Jonides, 1984; Egeth and Yantis, 1997). This shift of attention is often followed by a rapid or saccadic eye movement to bring central vision to bear on the change (Henderson and Hollingworth, 1999, 2003). If, however, the change is buried in a global visual transient such as a brief blank or a splash of distracting spots, the change in the local feature goes unnoticed (Rensink et al., 1997; O’Regan et al., 1999; Simons, 2000; Rensink, 2002). Attention that would normally have shifted to the focal change is confounded by changes throughout the scene caused by the global visual transient, rendering the change invisible (for a demonstration, see ftp://lsr-ftp.nei.nih.gov/web/jc/cb_demo.htm). This "change blindness" is not just a laboratory phenomenon, however. Large visual transients occur during every saccade, and changes occurring in the visual scene during saccadic eye movements often go unnoticed (O'Regan and Noe, 2001). Studies in humans have shown that attention can counter change blindness if directed, before the global visual transient occurs, to the feature that changes (Simons, 2000; Rensink, 2002).

This close relationship in normal vision between attention and saccades led us to develop a change blindness task in monkeys that we used to study brain mechanisms of visual attention. We did this in several steps (Cavanaugh and Wurtz, 2002, 2003).

Received Sept. 8, 2004; revised 0ct. 21, 2004; accepted 0ct. 22, 2004.

We thank Mitchell Smith, Nick Nichols, and Tom Ruffner for assistance with the experiments. We also thank our colleagues in the Laboratory for Sensorimotor Research at the National Eye Institute for helpful discussions and valuable comments.

Correspondence should be addressed to James Cavanaugh, Laboratory of Sensorimotor Research, National Eye Institute, National Institutes of Health, Building 49, Room 2A50, Bethesda, MD 20817. E-mail: jr@|lsr.nei.nih.gov. DOI:10.1523/JNEUROSCI.3724-04.2004

Copyright $\odot 2004$ Society for Neuroscience $\quad 0270-6474 / 04 / 2411236-08 \$ 15.00 / 0$
First, we developed a new change blindness task that required detecting changes in direction of visual motion. We recognized that change blindness for motion had two advantages: visual parameters for motion are well defined and separable (direction, speed), meaning we could isolate a single variable for change, and visual motion processing originates in areas of cerebral cortex that have been linked to visual attention by numerous studies (Treue and Maunsell, 1996, 1999; Seidemann and Newsome, 1999; Treue and Martinez Trujillo, 1999; Recanzone and Wurtz, 2000; Cook and Maunsell, 2002; Ghose and Maunsell, 2002; Martinez-Trujillo and Treue, 2002, 2004). Because the investigation of change blindness for motion is new, we first verified the effect in humans. Second, we ascertained the change blindness effect in monkeys for the first time by using the same motion detection paradigm we used in humans. We measured shifts of attention using the standard behavioral measures of visual attention used in human experiments: increased ability to detect the change and decreased reaction time to the change. Third, we used our newly developed task on monkeys to investigate whether the brain mechanisms used in selecting saccade targets might be the same mechanisms used to shift visual spatial attention. We did this by electrically stimulating a brainstem structure involved in the programming of saccadic eye movements, the superior colliculus (SC), to see if activation of this area counters change blindness in a manner consistent with a shift in visual spatial attention.

\section{Materials and Methods}

Change blindness task. The subjects' task (either human or monkey, $\mathrm{Ma}$ caca mulatta) was to detect a change in the direction of motion in one of three patches of random dot patterns back-projected onto a screen $58 \mathrm{~cm}$ in front of them by an LCD projector. Random dots were $0.3^{\circ}$ square and white against a gray background. Dot density was such that 5-7\% of the total stimulus area was covered with dots. Each dot had a lifetime of 167 msec and moved at $15 \% \mathrm{sec}$. Motion coherence was $100 \%$. Each trial 
began with the subject fixating on a spot of light in the center of the screen (see Fig. 1). Eye position was determined by an implanted scleral search coil for the monkeys and by an infrared eye-tracking device for the humans. Eye movements were digitized at $1000 \mathrm{~Hz}$ and stored at either 250 or $1000 \mathrm{~Hz}$. Eye position during fixation was required to remain within $1.5^{\circ}$ of the central point. After $500 \mathrm{msec}$ of fixation, on $50 \%$ of the trials a cue stimulus $\left(0.5^{\circ}\right.$ in diameter and centered on the future target location) indicated to the subject which patch would be the target (the patch in which the direction of the motion might later change). On the other half of the trials no cue appeared. The three patches, equally spaced around the central point, then appeared for 750-1500 msec. Motion in each patch was in a direction selected at random on each trial. The centers of the patches $\left(7.5-15.0^{\circ}\right.$ diameter) were placed between $8.5^{\circ}$ and $20^{\circ} \mathrm{ec}-$ centric from the fixation point. In preliminary experiments we determined that this particular combination of patch size and eccentricity provided a task that was neither too difficult nor too easy for the subjects (both human and monkey) to perform. The motion patches disappeared for $150 \mathrm{msec}$, causing a transient visual disruption (blank), followed by the reappearance of the motion patches with the direction of motion in the target patch changed on $\sim 65 \%$ of trials. Trials without blanks were randomly interleaved with blanked trials to make the overall task somewhat easier. Although trials without blanks accounted for typically $65 \%$ of total trials, they were not included in our analyses. That is, trials without blanks did not figure into our calculations of hits and false positives. The subject's task was to saccade to the target patch if it changed. On the $\sim 35 \%$ of trials in which no direction change occurred in the target, the correct response was to continue to fixate (catch trials). The direction of motion in the distractor patches never changed, but any patch could be the target. In psychophysical experiments, patch location was randomized among trials. Humans were rewarded after each correct trial by a short beep, and monkeys were rewarded with a drop of water. Monkeys were thoroughly trained on the task before commencing either the psychophysical or stimulation experiments. Although baseline performance could change from day to day, there was little systematic change in overall performance over the course of these experiments.

Note that this task differs from previous change blindness tasks for humans in that our subjects (human and monkey) were required to fixate until a change was seen, rather than able to view the image freely. Moreover, we restricted the change to a single attribute, direction of visual motion, which is encoded by cortical areas (MT, MST) in which it is known the activity of neurons is modulated when attention is directed within their receptive fields (Treue and Maunsell, 1996, 1999; Seidemann and Newsome, 1999; Treue and Martinez Trujillo, 1999; Recanzone and Wurtz, 2000; Cook and Maunsell, 2002; Ghose and Maunsell, 2002; Martinez-Trujillo and Treue, 2002, 2004).

The human subjects were an author (J.C.) and another member of the laboratory. Both had normal or corrected-to-normal vision. Experimental protocols used on humans were approved by the Institutional Review Board concerned with the use of human subjects.

Trials on which the subject made a saccade to the target patch when it changed (within a window of $\pm 3.5-4.5^{\circ}$ of the patch center) were hits. The proportion of hits was the number of hits out of the total number of trials on which the target did change. Saccades to a patch when there was no change in direction of motion in that patch (even if another patch did change) were considered false positives. Hits and false positives were calculated separately for different trial types (i.e., trials with a cue and trials without a cue). We assessed subject performance by plotting hits versus false positives and comparing these values for trials with and without the visual cue. We converted the differences between proportions (with and without a cue) into $z$-scores, representing the number of SDs by which the two distributions of proportions differed. To determine the significance of improvements in performance, we converted $z$-scores directly into significance levels. For example, significance levels of $0.0001,0.01,0.05,0.1$, and 0.25 are equivalent to $z$-scores of $3.72,2.33$, $1.64,1.28$, and 0.67 , respectively.

Because reaction times were not normally distributed, we used a nonparametric test for significance of differences between saccade latencies in the two conditions being compared (Wilcoxon rank-sum test).

Change blindness with stimulation. During stimulation experiments, the monkey again had to detect a change in direction within the target patch and make a saccade to that patch if it changed. In overlapping experiments, we placed one patch at the location in the visual field represented by the SC stimulation site. The remaining two patches were placed at the same eccentricity as the first, but evenly spaced around the fixation point. Target location was randomized by trial among the three positions. In this task, however, no visual cue was given. Instead, on 50\% of the trials on which the target was associated with the SC stimulation site, we stimulated the SC for $600 \mathrm{msec}$ around the time of the $150 \mathrm{msec}$ blank period before the change in motion direction (see Fig. $4 A$ ). Stimulation began $300 \mathrm{msec}$ before the blank and terminated $150 \mathrm{msec}$ after the stimulus patches reappeared. Direction changes occurred on $65 \%$ of trials. On the remaining $35 \%$ of trials, no direction change occurred in the target patch, and the correct response on these trials was to continue to fixate. We used a large number of these catch trials because we wanted to reduce any tendency for the monkey to make saccadic guesses. Also, because we were in a structure in which stronger stimulation evoked saccades, we wanted to allow many trials in which no eye movements were required to see whether stimulation itself would induce a saccade.

In another set of experiments at the same stimulation site, we stimulated the SC when one of the distractors overlapped the SC stimulation site. The target associated with stimulation in these nonoverlapping experiments was always located in the visual hemifield opposite that of the visual field representation of the collicular stimulation site and was always the same patch throughout an experiment. Overlapping and nonoverlapping experiments were always paired at the same stimulation site. Overlapping experiments were performed on different days than nonoverlapping experiments, but they were sometimes interleaved on alternate days. We had to end a series of experiments and abandon a stimulation site if either the stimulation threshold climbed too high or if the visual field location of the collicular stimulation site shifted because of small variations in electrode placement. Because of the finite number of days a stimulation site was viable, we sometimes were unable to obtain a full set of nonoverlapping data from a stimulation site.

We compared the frequency of the monkeys' hits and false positives on trials with SC stimulation to those without it. The proportions of hits and false positives in stimulation experiments were calculated separately for each patch location. Hits were saccades to a patch when it changed, and false positives were incorrect saccades to a patch out of the total number of trials on which that particular patch did not change. For nonoverlapping experiments, hits and false positives during stimulation trials were calculated for the target patch associated with stimulation trials, not the distractor patch overlapping the stimulation site. Calculating false positives separately for each patch location allowed us to carefully track not just whether the eyes moved during stimulation, but where they moved. We used the same $z$-score technique as for the psychophysical results to determine statistical significance of the effect of stimulation.

$S C$ recording and stimulation. In each of the two monkeys a cylinder was implanted over the SC (centered on midline and angled at $42^{\circ}$ so that the electrode advanced rostrally), and neurons were recorded extracellularly using single tungsten microelectrodes and standard amplification, spike discrimination, and computerized data collection procedures (Sommer and Wurtz, 2000). All procedures were approved by the Institute Animal Care and Use Committee and complied with Public Health Service Policy on the humane care and use of laboratory animals. After the SC had been located by single neuron recording, we used the same recording electrodes, but now with an impedance $<300,000 \Omega$ to stimulate the SC. To position these electrodes for stimulation, the microelectrode was advanced until it exited from a guide tube positioned several millimeters above the SC and then advanced until single neuron activity clearly indicated the visual responsiveness of the superficial layers of the SC. Then, as we advanced the electrode further, the SC was electrically stimulated for typically $300 \mathrm{msec}$ with a $200 \mathrm{~Hz}$ pulse train with biphasic pulses of $0.1 \mathrm{msec}$ in duration. As soon as stimulation at $60 \mu \mathrm{A}$ began to evoke saccades, the current strength was lowered and the electrode was advanced until a current of $<10 \mu \mathrm{A}$ evoked saccades $50 \%$ of the time. The region of the field to which the saccade was directed was noted and the center of one of the motion patches was placed at this point. We used a single magnitude of direction change in stimulation experiments, usu- 
ally $40^{\circ}$, as we determined that this made the experiment neither too difficult nor too easy for the monkeys.

After determining the location of the stimulation site by evoking saccades, we then reduced the frequency of stimulation from 200 to $70 \mathrm{~Hz}$ to activate the target area in the SC without evoking saccades. As reported previously by Glimcher and Sparks (1993), this lower frequency never evoked saccades even with a slight increase in current. We increased the current level past the threshold current, typically to $15 \mu \mathrm{A}$. This current level was then used for the rest of the experimental day. Interestingly, we achieved the best results when the stimulation current was low, because a low stimulation current followed from a low threshold current, which was determined by the relative excitability of the specific collicular stimulation site. In some experiments we left the electrode cemented into place for a number of daily recording sessions. In others we left the guide tube in place but reintroduced the electrode each day. Stimulation with the electrode newly introduced each day enabled us to consistently obtain the same approximate threshold current at a single stimulation site for all experiments performed at that site and produced the most consistent behavioral effects.

Data for each experiment were recorded over a period of three or more days, and we sometimes had to maintain a stimulation site for up to 2 weeks to obtain both overlapping and nonoverlapping data. The length of experiments was determined by the requirement for a large number of control trials and catch trials to insure that the location of the target remained unpredictable and that SC stimulation did not increase the frequency of saccades. For example, assume a prototypical overlapping experiment lasting $3 \mathrm{~d}$ in which the monkey performed 1000 trials each day. Of the 3000 total trials, only $1000(1 / 3)$ were directed at the target overlapping the stimulation site; the remainder were randomly interleaved controls to maintain the unpredictability of the target location. Of these 1000 trials directed at the stimulation site, $65 \%$ (typically) were randomly interleaved trials on which no blank occurred, to keep the monkey from getting discouraged with the more difficult blanked trials. Of these 350 remaining blanked trials, only half (175) were trials on which the SC was stimulated. This ratio $(175 / 3000$, or $<6 \%$ of trials) illustrates the necessity of the large numbers of trials over multiple days required for determining significance of the effect of stimulation while maintaining the unpredictability of the target.

\section{Results}

\section{Change blindness for motion}

In our change blindness task the subjects (two humans and two monkeys) began each trial by fixating on a spot in the center of the screen in front of them (see Materials and Methods). Three patches of random dot motion then appeared: one target and two distractors (Fig. 1). On $\sim 65 \%$ of trials, the direction of motion in

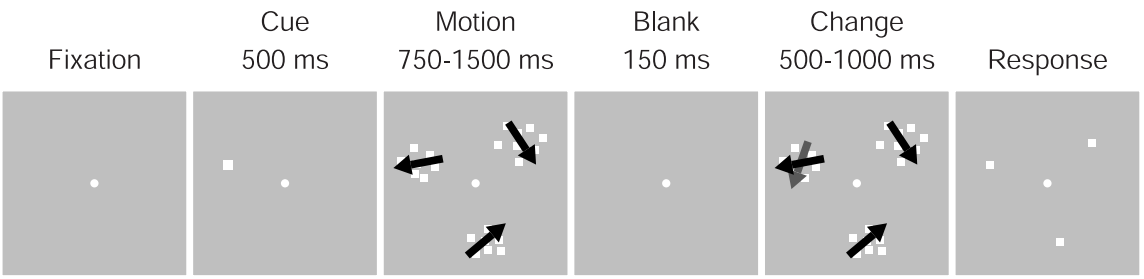

Figure 1. Change blindness for motion paradigm. After fixating, the subject was presented with three patches of random dot motion. On $50 \%$ of trials, the appearance of the patches was preceded by a cue, which was always valid for the location of the target. After $750-1500$ msec of motion, the patches disappeared for $150 \mathrm{msec}$ (blank) and then reappeared. $\mathrm{n} \sim 65 \%$ of trials, the direction of motion in the target patch had changed when the patches reappeared. After the reappearance of the patches, the subject was free to indicate whether the direction of motion in the target patch changed by making a saccade to the target patch or to remain fixating if no change was detected. Subjects were rewarded for correct saccades to the target when it changed or for remaining fixated if there was no change. The direction of motion never changed in the two distractor patches. In trials with no cue, there was no indication which patch was the target. The location of the target was randomized for each trial.
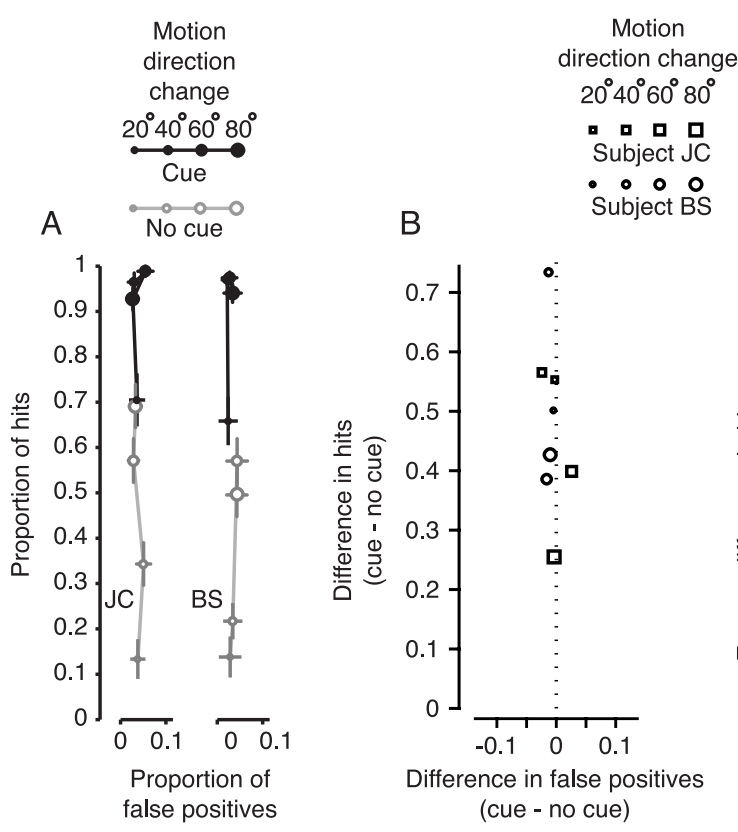

C
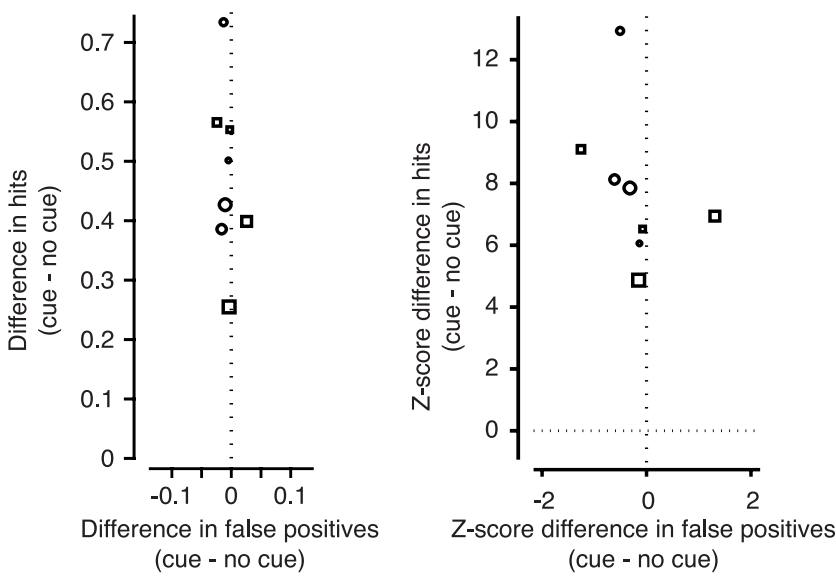

\section{Human psychophysics}

Figure 2. Change blindness for motion in humans. A, Change blindness task: absolute performance. Symbol size denotes the magnitude of the change in direction of motion. Filled symbols are from cued trials, whereas open symbols are from trials with no cue. Points are plotted for each magnitude of change in direction as proportion of hits versus proportion of false positives. Note that chance performance depended on both the proportion of trials on which the target changed and the number of targets. The proportion of hits expected from chance performance in these experiments would be $\sim 0.22$. The bars on each point are the SEs for proportion of hits (vertical) and false positives (horizontal). $B$, Change blindness task: change in performance. Symbol size still denotes the magnitude of the change in direction of motion, but symbol shape denotes subject. Points are plotted as the change in hits versus the change in false positives for each change in motion direction. Hits increased when the visual cue was presented. False positives changed very little. C, Change blindness task: change in $z$-score. Points are plotted as the change in hits versus the change in false positives for each change in motion direction in terms of $z$-score. Hits increased significantly $(z>3.72 ; p<$ $0.0001)$. False positives did not significantly change $(z<2.33 ; p>0.01)$.

the target patch changed. The subject indicated a change in the target by making a saccade to the target if it changed, and to continue fixating if it did not. The direction of motion in the distractor patches never changed, and the location of the target patch was randomized among trials. On half the trials, before the patches appeared, a visual cue indicated which patch was the target, whereas on the other half of the trials there was no such cue. To induce change blindness on cued and non-cued trials, we initiated a visual transient (all the patches briefly disappeared; the "blank" in Fig. 1) just when the direction of dot motion in the 


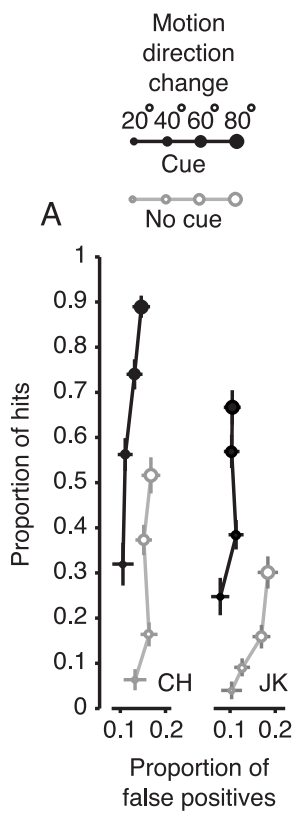

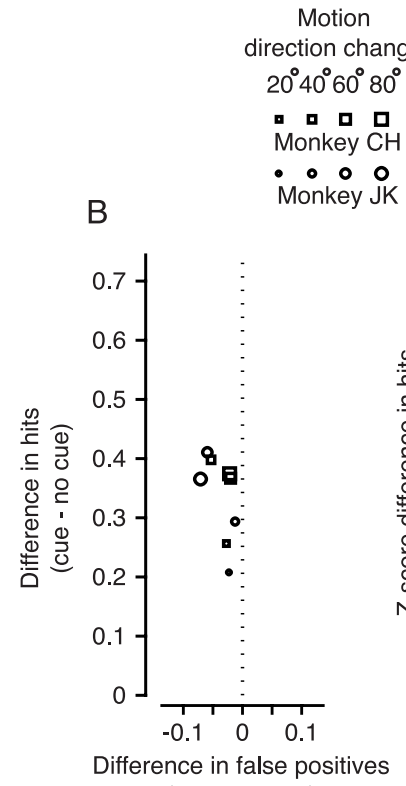

(cue - no cue)

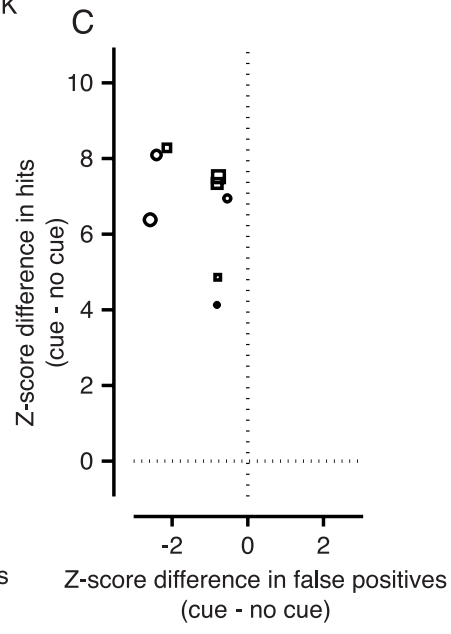

Monkey psychophysics
Figure 3. Change blindness in monkeys. $A$, Change blindness task: absolute performance. As in Figure 2, symbol size denotes the magnitude of the change in direction of motion. Filled symbols are from cued trials, whereas open symbols are from trials with no cue. Points are plotted for each magnitude of change in direction as proportion of hits versus proportion of false positives. Note that chance performance depended on both the proportion of trials on which the target changed and the number of targets. The proportion of hits expected from chance performance in these experiments would be $\sim 0.22$. The bars on each point are the SEs for proportion of hits (vertical) and false positives (horizontal). $B$, Change blindness task: change in performance. Symbol size still denotes the magnitude of the change in direction of motion, but symbol shape denotes subject. Points are plotted as the change in hits versus the change in false positives for each change in motion direction. As for human subjects, the attentional cue increased performance by significantly increasing the proportion of hits. C, Change blindness task: change in z-score. Points are plotted as the change in hits versus the change in false positives for each change in motion direction in terms of $z$-score. Hits increased significantly $(z>3.72 ; p<0.0001)$. False positives did not significantly change $(z<2.33 ; p>0.01)$ but occasionally approached significance (points to the far left).

target might change. To measure the influence of the attentional cue on change blindness we compared the subject's performance on trials without a cue with performance on trials with a cue.

Figure 2 shows the results for two human subjects. Figure $2 \mathrm{~A}$ shows the proportion of hits (saccades to the target when it changed) plotted against the proportion of false positives (saccades to any patch that did not change). Symbol size denotes the magnitude of the change in direction of dot motion: $20^{\circ}, 40^{\circ}, 60^{\circ}$, or $80^{\circ}$. Filled symbols represent performance on trials with a visual cue; open symbols show trials with no cue. When there was no cue, performance was typically poor, but accurate detection increased with the magnitude of the change in direction. When a cue was given (solid symbols), the proportion of hits was markedly better for each direction change. The magnitude of the change in hits when the cue was presented is plotted in Figure $2 B$ against the magnitude of the change in false positives. Symbol size still denotes the change in direction of dot motion, but now symbol shape indicates the subject (square for J.C., round for B.S.). The increase in hits afforded by presenting the cue is clearly visible. We converted the difference between the two proportions (with and without a cue) into a $z$-score (Fig. 2C), which allowed us to not only compare results across experiments, but also determine statistical significance of the differences. For each magnitude of direction change, the increase in the mean proportion of hits was significant (because $z>3.72, p<0.0001$; see Materials and Methods). There was no significant change in false positives $(z<2.33, p<0.01)$, apparently because of the requirement to remain fixating during the large number of catch trials in which no change occurred.

The cue also reduced the reaction time: for the $40^{\circ}$ change in direction of motion (the change we subsequently chose for our stimulation experiments), the mean reaction time decreased by $45 \mathrm{msec}$ from 455 msec ( $p<0.0001$; Wilcoxon rank-sum test). Thus, the human subjects performed better with the cue according to two standard measures of visual attention: better detection of stimulus change and reduced response latency.

\section{Change blindness in monkeys}

When we used the same task on two monkeys we observed similar results; the cue also significantly improved their performance (Fig. 3). Figure $3 A$ shows the absolute performance for monkeys on the change blindness task for each change in direction, which is similar to that for the human subjects in Figure $2 A$. The change in performance for hits and false positives is plotted in Figure $3 B$, and the significance of the differences is demonstrated as $z$-scores in Figure 3C. For each magnitude of direction change, the mean proportion of hits changed significantly (Fig. 3C) $(z>$ $3.72, p<0.0001)$. The change in the proportion of false positives was also small for monkeys, but occasionally approached significance (Fig. 3C, far left points) $(z<$ 2.33). The cue also reduced the mean reaction time (for the $40^{\circ}$ change) for the monkeys by 32 msec from $337 \mathrm{msec}$ ( $p<0.0001$; Wilcoxon rank-sum test). Like humans, the monkeys performed better with the cue in terms of improved detection of change and reduced reaction time, both standard measures of visual attention.

\section{SC stimulation and shift of attention}

We then used our change blindness task to test the hypothesis that common neuronal mechanisms underlie both the generation of saccades to one part of the visual field and shifts of attention to that same part of the visual field. We hypothesized that in this motor theory of attention (Rizzolatti, 1983; Sheliga et al., 1994; Moore et al., 2003), these two functions do not diverge until a point in the neuronal circuitry close to the actual motor neurons. Consequently, we targeted the visuotopic map in the intermediate layers of the SC, assuming that the point of divergence occurs after the SC. Some neurons in the intermediate layers of the SC closely tied to saccade generation (Sparks and HartwichYoung, 1989) also have prelude or buildup activity, shown to be related to selection of saccade target (Glimcher and Sparks, 1992; Dorris and Munoz, 1995; Basso and Wurtz, 1998; Bell et al., 2004). Such prelude activity occurs at the same time that neuronal activity in visual cortical areas is enhanced during attentional tasks (Reynolds and Desimone, 1999). It has been shown that this prelude activity is modulated when the monkey attends to a region of the visual field (Kustov and Robinson, 1996), and more recently that this modulation occurs only with a spatial cue for 
A

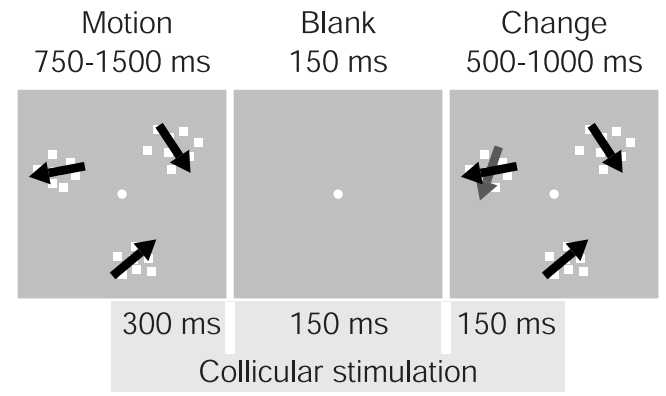

B

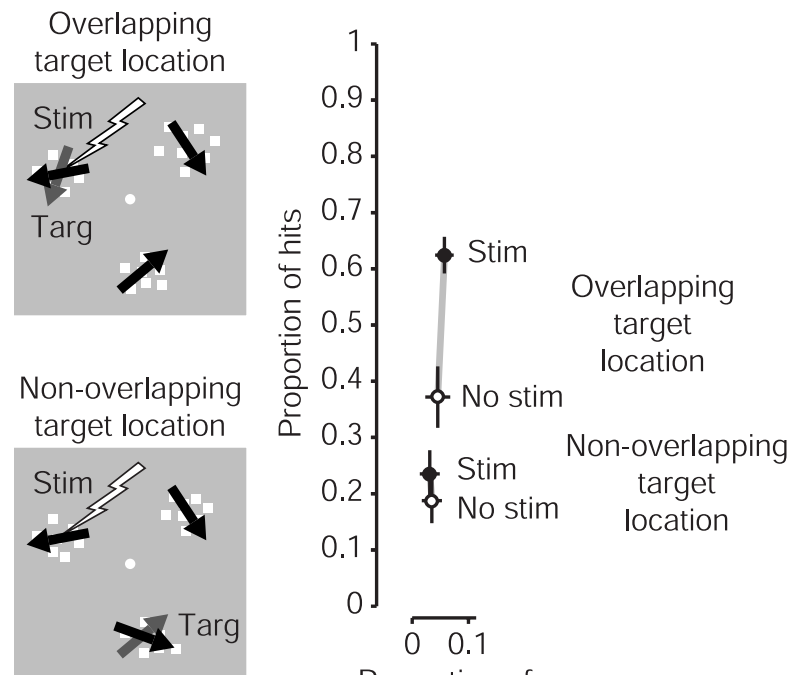

Proportion of

false positives

Figure 4. Superior colliculus stimulation: sample result. $A$, Time course of subthreshold collicular stimulation. Stimulation began $300 \mathrm{msec}$ before the blank period and continued for 600 msec, ending $150 \mathrm{msec}$ after the patches reappeared. Stimulation current and pulse frequency were well below levels required to elicit saccades. B, Overlapping and nonoverlapping experiment: sample results. In overlapping experiments, stimulation occurred when the target patch spatially overlapped the visual field location of the collicular stimulation site. In nonoverlapping experiments, we stimulated when one of the distractors spatially overlapped the collicular stimulation site. Change in motion direction for this experiment was $40^{\circ}$. The graph shows sample results from a single stimulation site. Note that each connected pair of points now represents a single target location. The ordinate and abscissa show proportions of hits and false positives, respectively. Open symbols show performance without stimulation, whereas filled symbols show the result with stimulation. Note that the only difference in these two experiments was the location of the target when stimulation occurred; the position of the stimulating electrode was the same. The difference in the open symbols (no stimulation) indicates the animal's pre-existing tendency to attend to one target over another. This tendency was not correlated with the size of the effect, because we achieved similar results whether the monkey had a predisposition to attend toward or away from the visual field representation of the SC stimulation site. When the target overlapped the site of collicular stimulation (overlapping), the proportion of hits increased greatly, whereas there was little change in the occurrence of false positives to this location. In the nonoverlapping experiment, neither hits nor false positives changed significantly.

that region (Ignashchenkova et al., 2004). Logically then, the buildup activity of these SC neurons might be directed not only to preparing for a saccade to one part of the visual field but also to providing an attentional signal that modulates the activity of visual cortical neurons related to the same part of the visual field.

We activated SC neurons related to one part of the visual field as the monkey performed the change blindness task (Fig. 4). Instead of providing a visual cue, we electrically stimulated the SC during the period when the change in direction might be occurring (Fig. 4A). We first identified the part of the visual field to which the SC was related by evoking saccadic eye movements with electrical stimulation. During our change blindness experiments, however, we reduced the stimulation frequency until it was too weak to elicit an eye movement (see Materials and Methods). At a sample stimulation site in one of the monkeys (Fig. $4 B)$, SC stimulation significantly increased the proportion of hits ( $p=0.0001$ ) when the target patch spatially overlapped the stimulation site. To determine whether the increase in hits from stimulation resulted from some general effect of stimulation such as arousal, we ran a separate set of trials in which we stimulated the SC $50 \%$ of the time only on those trials in which the target was one of the patches in the opposite visual hemifield and did not overlap the stimulation site. In addition to ruling out arousal from stimulation as mediating an increase in performance, the nonoverlapping trials would also rule out stimulation acting as a general cue, because the monkey should be able to learn to associate a $100 \%$ valid cue (the stimulation) with the associated nonoverlapping target. In this nonoverlapping case, however, we observed no significant increase in hit rate $(p=0.44)$. Furthermore, in neither the overlapping nor the nonoverlapping case did the false positive rate change for this stimulation site $(p>0.34)$, indicating that stimulation facilitated change detection and did not simply increase the frequency of saccades. The increase in only correct saccadic responses suggests that for this stimulation site, stimulating the SC countered change blindness in a spatially selective manner, akin to shifting covert attention with a visual cue.

The results of 23 experiments at 15 different stimulation sites in the SC of two monkeys showed modulation of performance to varying degrees. Figure 5 shows the effect of SC stimulation. We have plotted the differences in hit rates with and without stimulation against the same differences in false positives in Figure $5 \mathrm{~A}$. These same differences are expressed as $z$-scores in Figure $5 B$. Points above the dotted horizontal line at zero represent an increase in hit rate from SC stimulation, points to the right of the vertical dotted line at zero represent an increase in false positive rate. When the location of the target overlapped the representation of the SC stimulation site in the visual field (overlapping experiments, solid symbols), most points fell above the horizontal line, indicating an increase in hit rate. In contrast, when the target did not spatially overlap the SC stimulation site (nonoverlapping experiments, open symbols), most points fell below the horizontal line, indicating a decrease in hit rate. The example data shown in Figure $4 B$ are highlighted in Figure 5, $A$ and $B$, by circles.

Although not all changes in hit rate were significant by themselves ( $p \leq 0.01$ ) (Fig. 5, asterisks; 6/15 overlapping, 1/8 nonoverlapping), the mean increase in hit rate across overlapping experiments was significant $(9.2 \% ; p<0.0001)$. There was a slight decrease in the mean hit rate for nonoverlapping experiments, but this change was not highly significant $(-3.4 \%$; $p=$ $0.06)$. There was no significant change in the mean false positive rate for overlapping experiments $(-0.3 \% ; p=0.31)$, but the slight decrease in mean false positive rate for nonoverlapping experiments was significant $(-2.3 \% ; p=0.0009)$. In our stimulation experiments, the size of the effect from stimulation was not correlated with the animal's baseline performance.

Reaction time also changed with SC stimulation. For overlapping experiments (Fig. 6), mean reaction time decreased by 15 msec $(p=0.0001)$. For nonoverlapping experiments (data not shown), reaction time increased by $12 \mathrm{msec}(p=0.07)$, presumably because of SC stimulation shifting attention away from the target. 
A

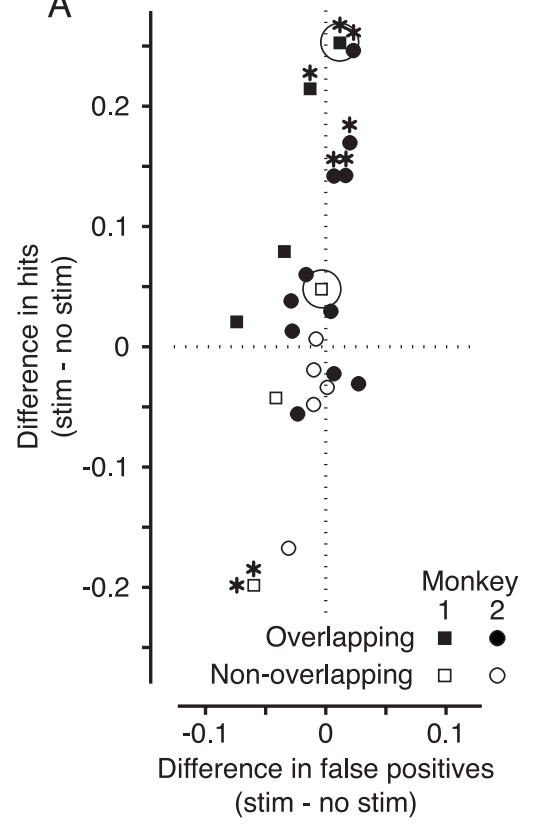

B

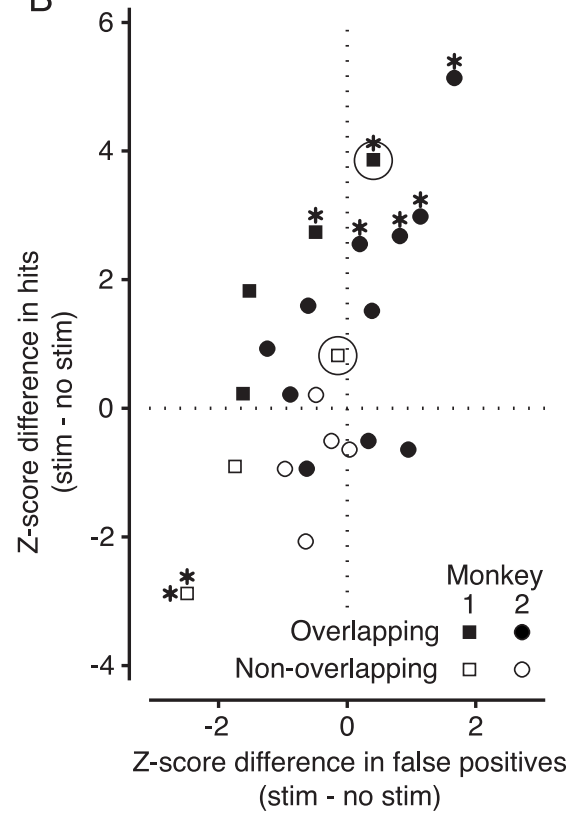

2002). We have shown the same effect for detection of changes in visual motion. We countered change blindness by shifting attention to the location of the motion change with a cue, and we quantified this shift of attention using two standard behavioral measures of attention: improved performance and decreased reaction time. When we had monkeys perform the same task, we showed that we could counter change blindness in the monkey according to the same measures of attention as in the human. By confirming that we could counter change blindness in the monkey by shifting attention to the location of a change in visual motion, we established a new model for investigating the neuronal mechanisms of visual spatial attention.

We turned our attention to the superior colliculus, hypothesizing that the SC sends signals not only through the brainstem for the generation of saccades (Sparks and Hartwich-Young, 1989) and the selection of saccade targets (Wurtz et al., 1980; Glimcher and Sparks, 1992; Basso and Wurtz, 1998; Horwitz and Newsome, 1999; Krauzlis and Dill, 2002; Port and Wurtz, 2003; McPeek and Keller, 2004), but also sends signals to the cerebral cortex for the facilitation of visual processing -the motor theory of attention. The improvements in performance and reaction time that we have shown in our change blindness task indicate that SC stimulation

Superior colliculus stimulation-population sults. A, Absolute change in performance. $A$ shows the ulation on change detection. Each point indicates results for one of 23 experiments performed at 15 different collicular stimulation sites in two monkeys. Values plotted are the differences between the stimulated and nonstimulated trials (stim - no stim) in each experiment, indicating how stimulation of the superior colliculus affected the monkey's performance on the change bir task. The ordinate represents the change in proportion of hits, whereas the abscissa shows the change in the proportion of false positives. Filled symbols show results from overlapping experiments, and open symbols are from nonoverlapping experiments. Positive values indicate that hits (or false positives) increased with stimulation. Significant changes $(p<0.01)$ in hit rate are shown by an asterisk above the symbols, whereas significant changes in false positive rate are denoted by an asterisk to the left. The results from the experiment in Figure $4 B$ are highlighted with circles. $B, Z$-score changes in performance. The changes shown in $A$ are replotted in $B$ as the $z$-scores of the differences. Significant values $(z>2.33 ; p<0.01)$ are indicated by asterisks as in $A$. Again, the results from the experiment in Figure $4 B$ are highlighted with circles.

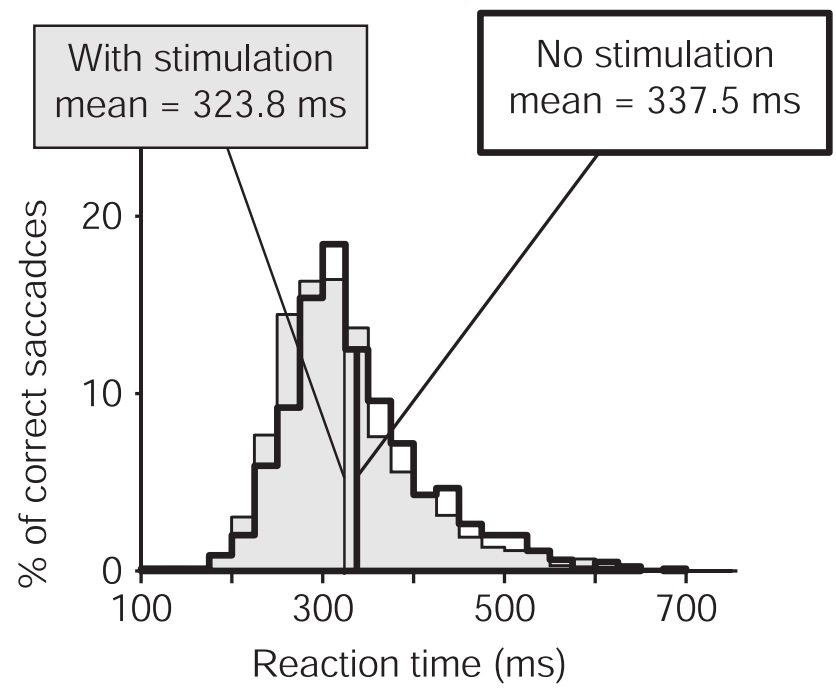

Figure 6. Superior colliculus stimulation: reaction times. Change in reaction time for overlapping experiments. Distributions of reaction times are shown for correct saccades from all overlapping experiments. Data from trials without stimulation are shown as the unshaded histogram ( $n=792)$, whereas the shaded histogram shows reaction times of correct saccadic responses from trials with collicular stimulation $(n=1060)$. Reaction time decreased significantly with stimulation ( $\mu=-15$ msec; $p<0.0001$; Wilcoxon rank-sum test).

\section{Discussion}

Change blindness in humans has previously has been demonstrated for changes in the content of natural scenes (Rensink, caused the same type of enhancement in performance as did a visual cue; a spatially selective increase in hits and reduction in reaction time. Although there are differences in the magnitudes of the changes between the psychophysical and stimulation experiments, differences one might expect even from two different types of visual cues, the fact remains that collicular stimulation caused the same type of significant improvements in performance as an attentional cue.

These results provide support for the motor theory of attention, specifically our hypothesis that the SC activity preceding the generation of saccadic eye movements to one part of the visual field also contributes a spatially selective attentional input for the enhanced visual processing seen in visual cortical areas. Such cortical enhancement would result from projections originating in the SC such as those conveying signals to frontal cortex (Sommer and Wurtz, 1998), or to cortical motion processing areas such as area MT, which is modified by SC stimulation (Muller et al., 2003).

Electrical stimulation, however, cannot be regarded as identical to normal neuronal activity within the SC. In addition to activating projections from the SC to cerebral cortex, the electrical stimulation also can produce antidromic activation of cortical areas projecting to the SC. Such activation would include the frontal eye field (FEF) because neurons in that region have prominent functional connections to the SC (Segraves and Goldberg, 1987; Sommer and Wurtz, 2000). In addition, previous experiments by Moore and Fallah $(2001,2004)$ have shown that stimulation of the FEF facilitates detection of targets during a visual search task consistent with a shift of visual attention. It therefore 
might be that our stimulation of the SC is actually activating FEF neurons (either antidromically or orthodromically), and the effect of SC stimulation acts via the FEF. Alternatively, stimulation of the FEF might produce attention effects because of its connections to the SC. Thus, stimulation of neither the SC nor the FEF can be regarded as affecting only that structure. Instead, such stimulation should be taken as indicating activation of a series of saccade related areas, and it is these areas that we believe contribute to the mechanisms underlying visual spatial attention.

As in the previous stimulation experiments related to attention (Moore and Fallah, 2004), another issue raised by the use of electrical stimulation is whether it simply produces a flash of light (a phosphene) in one part of the visual field. The question is essentially whether the stimulation produces a visual cue (a flash of light) or whether the stimulation directly produces a shift of attention. We think several factors argue that stimulation is not acting as a visual cue. If the stimulation were acting as a flash of light, we would expect a disruption of attention. The human literature on change blindness shows that stimuli presented at the time of a change in the visual scene in change blindness experiments diminishes the subject's ability to detect the change (Rensink et al., 1997; O'Regan et al., 1999; Simons, 2000; Rensink, 2002). Instead, we see an improvement in attention with stimulation at the time of the change. In addition, there is little evidence that stimulation of the intermediate layers of the SC produces a phosphene (Nashold, 1970), and even in the cerebral cortex phosphenes were seen with stimulation of occipital visual cortex but not outside of it (Brindley, 1982). The visual phosphene interpretation of stimulation cannot be rejected, but it does not seem the most likely consequence of SC stimulation, and is therefore a doubtful interpretation of our results. In any case, the question of stimulation producing a cue is amenable to experimental test. Shifting the time of stimulation earlier in the trial (closer to the time when the actual visual cue was given) should improve the shift of attention if a phosphene is acting as a visual cue. Additionally, stimulation of the superficial visual layers of the SC might be expected to yield an even stronger attention effect if the stimulation were producing a visual cue.

We believe that SC activity is associated with both endogenous and voluntary shifts of attention, which we used in our cued change blindness task, and the exogenous and involuntary shifts of attention, such as those occurring with sudden onset of an object. Both types of shifts are usually associated with saccades, and of course, the linking of the attentional shift and the saccade to the same part of the visual field is an integral part of our hypothesis. Even when a saccade is not made to the target, as in our catch trials, we know that the SC neurons have a buildup of activity (Munoz and Wurtz, 1995). It is this very buildup activity that we suggest would be relevant to facilitating visual processing in cerebral cortex. Ignashchenkova et al. (2004) have shown that this presaccadic delay activity was correlated with increased attentional performance in a spatially cued attention task. To further decouple the attentional shift from the actual saccade, we used a large number of catch trials during SC stimulation in which the subject was required to remain fixating. Thus, we had ample opportunity to evaluate whether the SC stimulation was simply producing more saccades (which would mean more false positives as well as more hits) rather than a selective shift of attention. False positives often decreased with stimulation, even in the nonoverlapping experiments, as one might even expect from a visual cue drawing attention away from the target. Because a saccade to any nonchanging patch was a false positive, we would have detected any tendency for stimulation to trigger saccades.
The low false positive rate on catch trials indicates that SC stimulation did not simply produce more saccades, but rather acted selectively to increase change detection.

Saccadic eye movements have long been regarded as a challenge to our perception of visual stability because they move the image falling on the retina several times per second (Yarbus, 1967). Recent experiments have shown that attention to specific features in the visual scene is critical for our stable perception despite these continual image shifts (Henderson and Hollingworth, 1999). Our experiments suggest that the same neuronal activity that prepares for generating the eye movements that challenge the stability of our perception also contributes to the shifts of visual attention that help unify our perception.

\section{References}

Basso MA, Wurtz RH (1998) Modulation of neuronal activity in superior colliculus by changes in target probability. J Neurosci 18:7519-7534.

Bell AH, Fecteau JH, Munoz DP (2004) Using auditory and visual stimuli to investigate the behavioral and neuronal consequences of reflexive covert orienting. J Neurophysiol 91:2172-2184.

Brindley GS (1982) Effects of electrical stimulation of the visual cortex. Hum Neurobiol 1:281-283.

Cavanaugh J, Wurtz R (2002) Change-blindness for motion in macaque monkey. J Vision 2:16a.

Cavanaugh JR, Wurtz RH (2003) Detection of changes in direction of motion in a visual attention task is enhanced by stimulation of the superior colliculus. Soc Neurosci Abstr 29:767.4.

Cook EP, Maunsell JHR (2002) Attentional modulation of behavioral performance and neuronal responses in middle temporal and ventral intraparietal areas of macaque monkey. J Neurosci 22:1994-2004.

Dorris MC, Munoz DP (1995) A neural correlate of the gap effect on saccadic reaction times in monkey. J Neurophysiol 73:2558-2562.

Egeth HE, Yantis S (1997) Visual attention: control, representation, and time course. Annu Rev Psychol 48:269-297.

Ghose GM, Maunsell JHR (2002) Attentional modulation in visual cortex depends on task timing. Nature 419:616-620.

Glimcher PW, Sparks DL (1992) Movement selection in advance of action in the superior colliculus. Nature 355:542-545.

Glimcher PW, Sparks DL (1993) Effects of low-frequency stimulation of the superior colliculus on spontaneous and visually guided saccades. J Neurophysiol 69:953-964.

Henderson JM, Hollingworth A (1999) High-level scene perception. Annu Rev Psychol 50:243-271.

Henderson JM, Hollingworth A (2003) Eye movements and visual memory: detecting changes to saccade targets in scenes. Percept Psychophys 65:58-71.

Horwitz GD, Newsome WT (1999) Separate signals for target selection and movement specification in the superior colliculus. Science 284:1158-1161.

Ignashchenkova A, Dicke PW, Haarmeier T, Thier P (2004) Neuronspecific contribution of the superior colliculus to overt and covert shifts of attention. Nat Neurosci 7:56-64.

Krauzlis RJ, Dill N (2002) Neural correlates of target choice for pursuit and saccades in the primate superior colliculus. Neuron 35:355-363.

Kustov AA, Robinson DL (1996) Shared neural control of attentional shifts and eye movements. Nature 384:74-77.

Martinez-Trujillo JC, Treue S (2002) Attentional modulation strength in cortical area MT depends on stimulus contrast. Neuron 35:365-370.

Martinez-Trujillo JC, Treue S (2004) Feature-based attention increases the selectivity of population responses in primate visual cortex. Curr Biol 14:744-751.

McPeek RM, Keller EL (2004) Deficits in saccade target selection after inactivation of superior colliculus. Nat Neurosci 7:757-763.

Moore T, Fallah M (2001) Control of eye movements and spatial attention. Proc Natl Acad Sci USA 98:1273-1276.

Moore T, Fallah M (2004) Microstimulation of the frontal eye field and its effects on covert spatial attention. J Neurophysiol 91:152-162.

Moore T, Armstrong KM, Fallah M (2003) Visuomotor origins of covert spatial attention. Neuron 40:671-683.

Muller JR, Philiastides MG, Newsome WT (2003) Subthreshold electrical 
stimulation of the superior colliculus (SC) modulates activity in the middle temporal visual area (MT). Soc Neurosci Abstr 29:767.3.

Munoz DP, Wurtz RH (1995) Saccade-related activity in monkey superior colliculus. I. Characteristics of burst and buildup cells. J Neurophysiol 73:2313-2333.

Nashold BS (1970) Phosphenes resulting from stimulation of the midbrain of man. Arch Opthal 84:433-435.

O'Regan JK, Noe A (2001) A sensorimotor account of vision and visual consciousness. Behav Brain Sci 24:939-973; discussion 973-1031.

O'Regan JK, Rensink RA, Clark JJ (1999) Change-blindness as a result of "mudsplashes". Nature 398:34.

Port NL, Wurtz RH (2003) Sequential activity of simultaneously recorded neurons in the superior colliculus during curved saccades. J Neurophysiol 90:1887-1903.

Recanzone GH, Wurtz RH (2000) Effects of attention on MT and MST neuronal activity during pursuit initiation. J Neurophysiol 83:777-790.

Rensink RA (2002) Change detection. Annu Rev Psychol 53:245-277.

Rensink RA, O’Regan JK, Clark JJ (1997) To see or not to see: the need for attention to perceive changes in scenes. Psychol Sci 8:368-373.

Reynolds JH, Desimone R (1999) The role of neural mechanisms of attention in solving the binding problem. Neuron 24:19-29:111-125.

Rizzolatti G (1983) Mechanisms of selective attention in mammals. In: Advances in vertebrate neuroethology (Ewert J-P, Capranica R, Ingle DJ, eds), pp 261-297. London: Plenum.

Segraves MA, Goldberg ME (1987) Functional properties of corticotectal neurons in the monkey's frontal eye field. J Neurophysiol 58:1387-1419.

Seidemann E, Newsome WT (1999) Effect of spatial attention on the responses of area MT neurons. J Neurophysiol 81:1783-1794.
Sheliga BM, Riggio L, Rizzolatti G (1994) Orienting of attention and eye movements. Exp Brain Res 98:507-522.

Simons DJ (2000) Attentional capture and inattentional blindness. Trends Cogn Sci 4:147-155.

Sommer MA, Wurtz RH (1998) Frontal eye field neurons orthodromically activated from the superior colliculus. J Neurophysiol 80:3331-3335.

Sommer MA, Wurtz RH (2000) Composition and topographic organization of signals sent from the frontal eye field to the superior colliculus. J Neurophysiol 83:1979-2001.

Sparks DL, Hartwich-Young R (1989) The deep layers of the superior colliculus. In: The neurobiology of saccadic eye movements, reviews of oculomotor research, Vol III (Wurtz RH, Goldberg ME, eds), pp 213-256. Amsterdam: Elsevier.

Treue S, Maunsell JH (1996) Attentional modulation of visual motion processing in cortical areas MT and MST. Nature 382:539-541.

Treue S, Maunsell JH (1999) Effects of attention on the processing of motion in macaque middle temporal and medial superior temporal visual cortical areas. J Neurosci 19:7591-7602.

Treue S, Martinez Trujillo JC (1999) Feature-based attention influences motion processing gain in macaque visual cortex. Nature 399:575-579.

Wurtz RH, Goldberg ME, Robinson DL (1980) Behavioral modulation of visual responses in the monkey: stimulus selection for attention and movement. In: Progress in psychobiology and physiological psychology (Sprague JM, Epstein AN, eds), pp 43-83. New York: Academic.

Yantis S, Jonides J (1984) Abrupt visual onsets and selective attention: evidence from visual search. J Exp Psychol Hum Percept Perform 10:601-621.

Yarbus AL (1967) Eye movements and vision. New York: Plenum. 